\title{
Influence of concurrent capecitabine based chemoradiotherapy with bevacizumab on the survival rate, late toxicity and health-related quality of life in locally advanced rectal cancer: a prospective phase II CRAB trial
}

\author{
Vaneja Velenik ${ }^{1,5}$, Vesna Zadnik ${ }^{2,5}$, Mirko Omejc ${ }^{3,5}$, Jan Grosek ${ }^{3,5}$, Mojca Tuta ${ }^{4,5}$ \\ ${ }^{1}$ Division of Radiotherapy, Institute of Oncology, Ljubljana, Slovenia \\ ${ }^{2}$ Division of Epidemiology, Institute of Oncology, Ljubljana, Slovenia \\ ${ }^{3}$ Division of Surgery, University Medical Center Ljubljana, Ljubljana, Slovenia \\ ${ }^{4}$ Division of Radiology, Institute of Oncology, Ljubljana, Slovenia \\ ${ }^{5}$ Medical Faculty, University of Ljubljana, 1000 Ljubljana
}

Radiol Oncol 2020; 54(4): 461-469.

Received 1 March 2020

Accepted 13 June 2020

Correspondence to: Assoc. prof. Vaneja Velenik, M.D., Ph.D., Institute of Oncology Ljubljana, Zaloška cesta 2, SI-1000 Ljubljana, Slovenia. E-mail: vvelenik@onko-i.si

Disclosure: No potential conflicts of interest were disclosed.

Background. Few studies reported early results on efficacy, toxicity of combined modality treatment for locally advanced rectal cancer (LARC) by adding bevacizumab to preoperative chemoradiotherapy, but long-term data on survival, and late complications are lacking. Further, none of the studies reported on the assessment of quality of life (QOL).

Patients and methods. After more than 5 years of follow-up, we updated the results of our previous phase II trial in 61 patients with LARC treated with neoadjuvant capecitabine, radiotherapy and bevacizumab (CRAB study) before surgery and adjuvant chemotherapy. Secondary endpoints of updated analysis were local control (LC), disease free (DFS) and overall survival (OS), late toxicity and longitudinal health related QOL (before starting the treatment and one year after the treatment) with questionnaire EORTC QLQ-C30 and EORTC QLQ-CR38.

Results. Median follow-up was 67 months. During the follow-up period, 16 patients $(26.7 \%)$ died. The 5-year OS, DFS and LC rate were $72.2 \%, 70 \%$ and $92.4 \%$. Patients with pathological positive nodes or pathological T3-4 tumors had significantly worse survival than patients with pathological negative nodes or T0-2 tumors. Nine patients (14.8\%) developed grade ${ }^{3} 3$ late complications of combined modality treatment, first event 12 months and last 87 months after operation (median time 48 months). Based on EORTC QLQ-C30 scores one year after treatment there were no significant changes in global QOL and three symptoms (pain, insomnia and diarrhea), but physical and social functioning significantly decreased. Based on QLQ-CR38 scores body image scores significantly increase, problems with weight loss significantly decrease, but sexual dysfunction in men and chemotherapy side effects significantly increase. Conclusions. Patients with LARC and high risk factors, such as positive pathological lymph nodes and high pathological T stage, deserve more aggressive treatment in the light of improving long-term survival results. Patients after multimodality treatment should be given greater attention to the regulation of individual aspects of quality of life and the occurrence of late side effects.

Key words: rectal cancer; bevacizumab; preoperative chemoradiotherapy 


\section{Introduction}

Colorectal cancer (CRC) represents a major public health problem in developed countries, especially in parts of Europe (Hungary, Slovenia, Slovakia, the Netherlands and Norway). ${ }^{1}$ In Slovenia CRC is most frequently diagnosed among age group 50-74. ${ }^{2}$ The standard treatment for LARC consists of capecitabine-based chemoradiation (CRT) followed by counseling surgery and adjuvant chemotherapy (ChT). Advances in multimodality treatments have significantly reduced 5-year local recurrence rates to less than $10 \%$ but this fact is not reflected in better survival., ${ }^{3,4}$ High rate of distant metastases $(30 \%$ at 10 years) represents the main problem in achieving even better results of rectal cancer treatment. ${ }^{5}$ Thus, to achieve better control of systemic disease and consequently better survival, intensified systemic therapy is warranted.

The main guideline in developing the most optimal rectal cancer treatment regimen is elimination of subclinical micrometastases or/and interruption of the metastatic cascade. Angiogenesis plays a significant role in tumor growth, invasion and metastasis. The benefit of antiangiogenic inhibitors on better survival is already known in the treatment of metastatic colorectal cancer. ${ }^{6}$ Pre-clinical experiments in a variety of tumor models have shown encouraging results with the combination of antiangiogenic strategies with cytotoxic agents such as chemotherapy, ionizing radiation, or both in rectal cancer. ${ }^{78}$ Although bevacizumab, a recombinant humanized monoclonal antibody against vascular endothelial cell growth factor (VEGF), is widely tested in the preoperative treatment of LARC, there are only few studies evaluating survival benefit.9-11 Consequently, safety and efficacy of adding bevacizumab in the preoperative treatment of LARC remain unclear.

Significant progress in various approaches to rectal cancer treatment has led to the fact that longterm results and assessment of a patient's quality of life (QOL) has become increasingly important for offering patient optimal treatment. The European Organization for Research and Treatment of Cancer (EORTC) Quality of Life Questionnaire (QLQ-C30) and its tumor-specific 38-item questionnaire module Quality of Life Questionnaire Colorectal Cancer Module (QLQ-CR38), that later got its successor QLQ-CR29 with revised and fewer questions, were the first questionnaires introduced specifically for CRC. ${ }^{12-14}$ In addition to these QOL assessment tools for CRC, the Functional Assessment of Cancer Therapy-Colorectal (FACT-C) is also widely used. ${ }^{13}$
The FACT-C places a larger emphasis on emotional aspects of QOL while the QLQ-CR38 and QLQCR29 have greater dominance in the assessment of disease- and treatment-related symptoms. ${ }^{13}$

Our phase II trial was originally designed to determine the pathologic complete response rate of CRT and bevacizumab as a part of a combined modality approach. This report includes the long-term outcome, late complications and health related QOL of patients treated in CRAB study.

\section{Patients and methods}

The trial design, eligibility criteria, treatment and assessments have been published previously in detail. ${ }^{15}$ All patients provided signed informed consent based on international standards. The study was approved by the National Medical Ethics Committee of the Republic of Slovenia (Number 173/07/08) and was in agreement with the Declaration of Helsinki. It was registered in the ClinicalTrials.gov database (NCT 00842686).

\section{Patient selection}

In brief, patients with histologically proven stage II/III adenocarcinoma of the rectum within $15 \mathrm{~cm}$ of the anal verge and without contraindications for ChT or targeted agents were included. Local extend of the disease was determined by magnetic resonance imaging.

\section{Treatment}

Radiotherapy (RT) was delivered using three-dimensional conformal computed tomography (CT)based treatment planning. Four-field box technique with all fields treated daily was used. Patients received 45 Gy to the pelvis plus 5.4 Gy as a boost to the primary tumor in 1.8 Gy over 5.5 weeks. Capecitabine $825 \mathrm{mg} / \mathrm{m}^{2}$ twice daily was administered concomitantly continuously throughout of RT without interruptions on weekends. Patients received bevacizumab intravenously at a dose 5 $\mathrm{mg} / \mathrm{kg} 14$ days prior and on days 1, 15 and 29 during chemoradiotherapy (CRT). Resection (low anterior resection or abdominoperineal amputation with total mesorectal excision) was performed 6-8 weeks after the completion of CRT. Patients with histopathological $\mathrm{R} 0$ resection received 6 cycles of adjuvant chemotherapy with capecitabine, while in those with R1 additional 2 cycles were given. Pathologic response after CRT with bevacizumab 
was determined according to Dworak tumor regression grade (TRG) system. The pathological complete response (pCR) was defined as TRG 4, meaning no tumor cells in surgical specimen.

\section{Statistical methods}

Results for primary endpoint of this prospective phase II study, the pCR rate, and on some secondary endpoints (pathological response rate, rate of sphincter-sparing surgical procedure, radical resection rate, acute and perioperative toxicity of combined modality treatment) have been reported previously. ${ }^{15}$ Here we report results on other secondary endpoints of this updated analysis: local control (LC), disease free (DFS) recurrence-free (RFS) and overall survival (OS), late toxicity and health related QOL. Survival rates were calculated using Kaplan-Meier technique. All time intervals were calculating from the date of inclusion. The end day for LC was the date of last follow-up or recurrence in the pelvis; for OS the date of last follow up or death from any cause; for DFS the date of relapse, secondary cancer, death for any cause or the last follow-up.

Separate analysis was performed for health related QLQ applying the questionnaires launched by the EORTC. For this study the core questionnaire EORTC QLQ-C3016 adjoined with the colorectal module EORTC QLQ-CR38 ${ }^{12}$ was delivered to the patients twice: before starting the treatment and one year after the treatment was finished. The EORTC QLQ-C30 is a questionnaire assessing individual HRQL during the previous week. The EORTC QLQ-C30 has 30 items and is divided into five function scales (physical, role, cognitive, emotional and social functions); three symptom scales (fatigue, pain, nausea or vomiting) and one global health-status/quality of life dimension. The six single items address specific symptoms: dyspnoea, appetite loss, insomnia, constipation, diarrhoea and a question addressing the financial impact of the disease. The EORTC QLQ-CR38 has 38 questions and is divided into four functional and seven scales of symptoms/problems. The answers recorded by the questionnaires were transformed into dimensions ranged 0-100 according to the EORTC scoring instructions. ${ }^{17}$ For functional scales and single items higher scores represent a higher level of functioning, but for symptom scales and single items, a higher score represents a higher level of symptoms. To examine the statistically significant changes in QLQ scores over time the Wilcoxon signed-rank test was applied.
All statistical analysis was performed using the SPSS statistical software package, version 24 (SPSS Inc, Chicago, IL, USA). Values of $\mathrm{p}<0.05$ were considered statistically significant.

\section{Results}

Between February 2009 and March 2010, 61 patients entered the study protocol at our institution. The detailed characteristics of the patients have been presented previously. ${ }^{15}$ Baseline assessment included complete history, physical examination, full blood count, serum biochemistry, carcinoembryonic antigen, chest radiography, ultrasonography and/or computed tomography (CT) scan of the whole abdomen. Twelve pts (19.7\%) presented with stage II and all other with stage III of disease. In 28 patients (45.9\%) the tumor invaded the mesorectal fascia. Radical resection was achieved in 57 pts (95\%). Sixty patients were eligible for efficacy analysis. TRG 4 (pCR) was recorded in 8 pts $(13.3 \%)$ and TRG 3 in 9 pts (15\%). Fifty-one pts (83.6\%) received capecitabine postoperatively. An intention-to-treat analysis was performed on 60/61 pts as one was misdiagnosed. Median follow-up was 67 months (range, 7 to 79 months). During the follow-up period, 16 patients $(26.7 \%)$ died. A total of $13(21.7 \%)$ of these deaths were a consequence of rectal cancer progression and the remaining 3 due to unrelated causes.

\section{Survival analysis}

The 5-year OS was $72.2 \%$ (95\% CI $58.2-84$ ). Recurrences were observed in 14 patients (23.3\%) and in one secondary cancer occurred. The latest distant recurrence was observed 54 months after the operation. The 5-years recurrence-free survival and DFS were $75.6 \%$ (95\% CI $64.6-81.3)$ and $70 \%$ (95\% CI 58.5-81.5), respectively. Local recurrence as any component of first failure occurred in 4 patients $(6.7 \%)$, with isolated local recurrence in 1 (1.7\%). The 5-year LC rate was $92.4 \%$ (95\% CI 85.499.4). Figure 1 illustrates OS, DFS and recurrencefree survival and $L C$ of patients treated in $C R A B$ trial.

Based on the Cox proportional hazards regression model, there were no significant association between OS or DFS and gender, age, performance status, cT, cN, TRG and adjuvant ChT. Patients with pathological positive nodes or pathological T3-4 tumors had significantly worse survival than 
TABLE 1. Overall survival (OS) and disease free survival (DSF) according to pTumor and pNodal stage on univariate analysis

\begin{tabular}{|c|c|c|c|c|}
\hline Factor & os & & DFS & \\
\hline \multicolumn{5}{|c|}{ pTumor stage } \\
\hline рT0-2 & $85 \%$ & & $85.7 \%$ & \\
\hline рT3-4 & $60.9 \%$ & $p=0.043$ & $61.8 \%$ & $p=0.044$ \\
\hline \multicolumn{5}{|c|}{ pNodal stage } \\
\hline pNO & $81 \%$ & & $81 \%$ & \\
\hline $\mathrm{pN}+$ & $37.5 \%$ & & $41.7 \%$ & $p=0.003$ \\
\hline
\end{tabular}
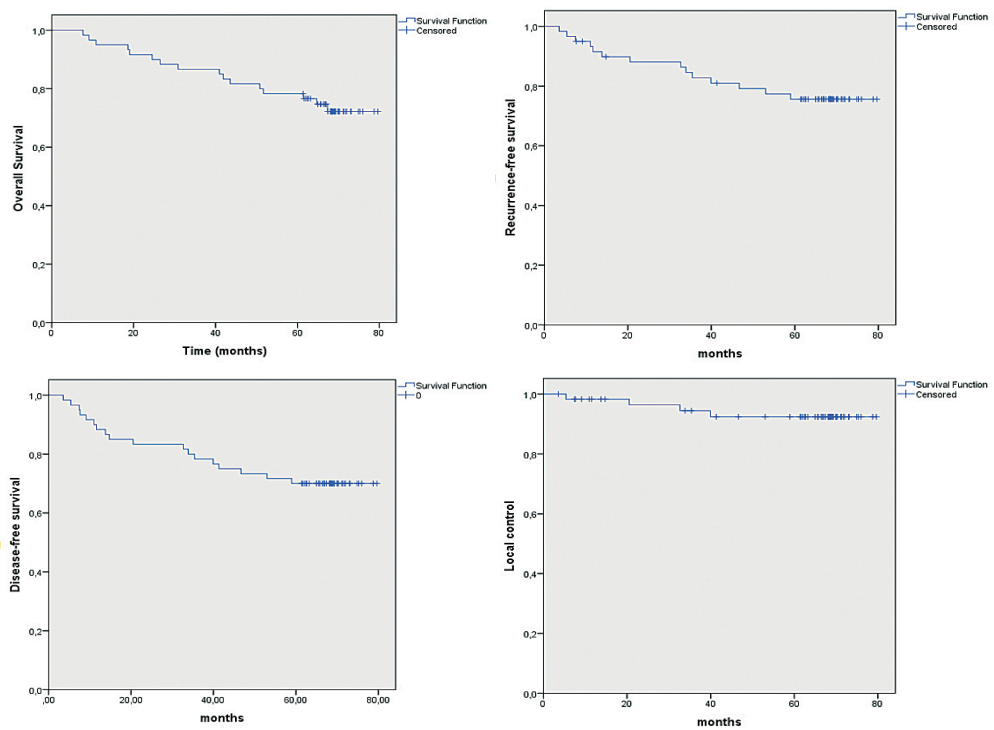

FIGURE 1. Overall survival (OS), disease free survival (DSF), recurrence-free survival and local control (LC) of patients treated in CRAB trial.
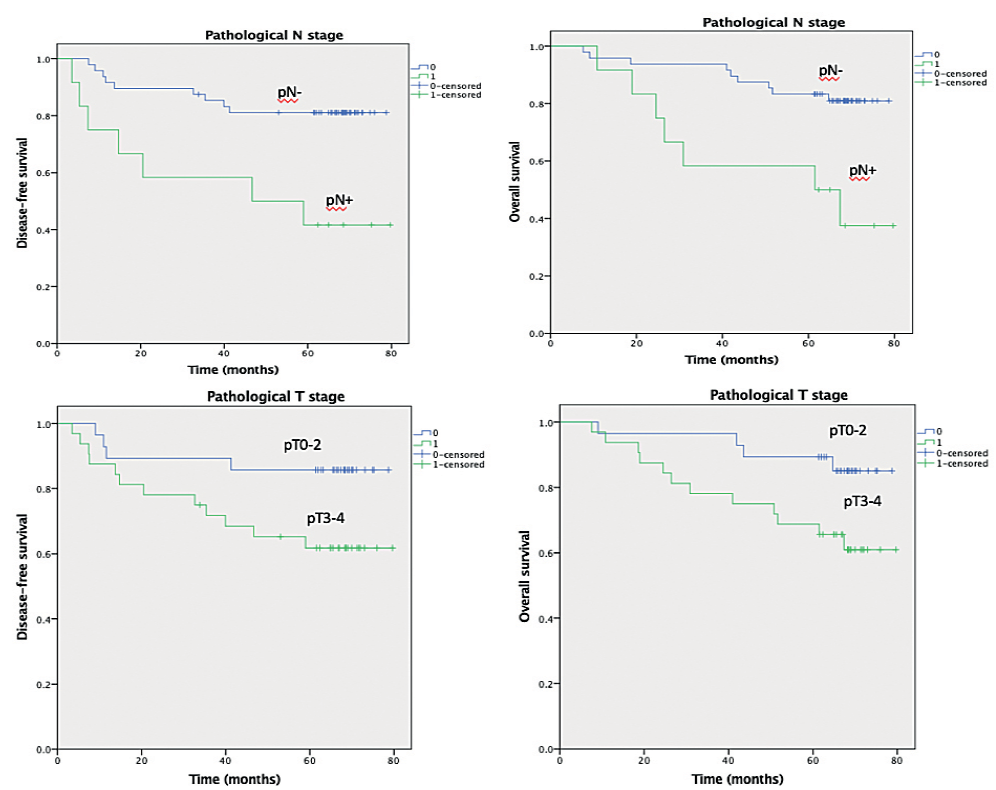

FIGURE 2. Prognostic significance of pathological nodal stage (pN) and tumor stage (PT) on 5-year disease free survival (DFS) and overall survival (OS). patients with pathological negative nodes or T0-2 tumor (Table 1 and Figure 2).

\section{Late complications}

Nine patients $(14.8 \%)$ developed grade 3 late complications of combined modality treatment (Common Terminology Criteria for Adverse Events version 4.0) for which hospitalization and/or urgent intervention was needed. We recorded the first event 12 months and the last 87 months after the operation with the median time to occurrence of 48 months. All these complications, time to occurrence after surgery of primary cancer and procedures needed are presented in Table 2. Among patients without mentioned complications, we observed permanent defecation complications in 11: constipation in 4, defecation urgency in 3 and fecal incontinence in 4 colostomy free patients. Two patients with stoma experienced permanent urinary complications: 1 incompleted bladder emptying and 1 urinary incontinence.

\section{Health related quality of life}

The results of the health related QOL analysis is summarized in Table 3. One year after treatment there were no significant changes in global quality of life, however the physical and social functioning significantly decreased. On the other hand, there was a significant decrease in three symptoms: pain, insomnia and diarrhea.

Based on QLQ-CR38 scores there were no significant change on three functional scales (i.e. future perspectives, sexual functioning and enjoyment), but the body image scores significantly increase. One year after treatment has been completed our patients reported significantly less problems with weight loss, but there was a significant increase in ChT side effects (dry mouth, thin or lifeless hair and different taste) and sexual dysfunction in men.

\section{Discussion}

The final results of CRAB study show that preoperative CRT with bevacizumab and capecitabine is feasible with good compliance and acceptable toxicity. ${ }^{15}$ The pCR rate of $13 \%$ was similar to an earlier phase II study by our group examining neoadjuvant single-agent capecitabine plus RT in LARC. ${ }^{18}$

Direct comparison of long term results could only be possible with the study from Gasparini et 
TABLE 2. Late grade ${ }^{3} 3$ adverse events in CRAB trial

\begin{tabular}{|c|c|c|c|}
\hline Event & $\mathbf{N}$ & $\begin{array}{l}\text { Time to appearance } \\
\text { (after the operation) }\end{array}$ & Procedure \\
\hline Fistula rectovaginalis & $\begin{array}{l}1 \\
1\end{array}$ & $\begin{array}{l}12 \text { months } \\
31 \text { months }\end{array}$ & $\begin{array}{c}\text { Hartman operation } \\
\text { Abdominoperineal excision }\end{array}$ \\
\hline Fistula enteroperinealis & 1 & 74 months & $\begin{array}{c}\text { No action due to local and distant progression of } \\
\text { the disease }\end{array}$ \\
\hline Fistula enteroglutealis & 1 & 54 months & Incision, drain \\
\hline Fistula uretroperinealis & 1 & 87 months & Conservative \\
\hline Fistula enteroperinealis & 1 & 54 months & No action due to poor performance status \\
\hline Abscessus perinealis & 1 & 48 months & Incision, drain \\
\hline Abscessus presacralis & 1 & 36 months & Incision, transversostomy \\
\hline Stenosis ureteri bill. & 1 & 43 months & J splint bill \\
\hline
\end{tabular}

$a l$, as they reported comparable characteristics of patients and the same treatment regimen (neoadjuvant bevacizumab $5 \mathrm{mg} / \mathrm{kg}$ on days -14, and 1, 15, 29, and concomitant CRT with capecitabine 825/ $\mathrm{mg} / \mathrm{m}^{2} / \mathrm{bid}$ with total radiation dose of $50.4 \mathrm{~Gy}$ ), but such data were not published. ${ }^{19}$ Only one study from Willett et al. reported 5-years survival data for LARC, treated with concurrent bevacizumab and preoperative capecitabine-based CRT. ${ }^{10}$ In comparison to our study Willet et al. reported comparable pCR rate of $16 \%$, although their study had slightly different treatment regimen (neoadjuvant bevacizumab $5-10 \mathrm{mg} / \mathrm{kg}$ on days $1,8,15,22$ and concomitant CRT with 4 cycles of fluorouracil 225 $\mathrm{mg} / \mathrm{m}^{2} / 24 \mathrm{~h}$ with total radiation dose of $50.4 \mathrm{~Gy}$ ). Additionally, our is the largest study on the long term efficacy and the only one evaluating late toxicity for neoadjuvant bevacizumab in LARC (Willet et al. vs. CRAB, 32 patients vs. 61 patients). ${ }^{10}$ To our knowledge our study is one of the first evaluating longitudinal health related QOL of rectal cancer patients after combined modality treatment and the only one with bevacizumab in this specific group of patients. ${ }^{20}$

\section{Survival}

Compared to our study, Willett et al. reported better 5-year LC rate $(92,4 \%$ vs. $100 \%)$ and OS $(72.2 \%$ vs. $100 \%) .{ }^{10}$ However, no difference in 5 -year DFS (Willett et al. vs. CRAB, $75 \%$ vs. $75.6 \%$ ) was observed. Pathologic complete response rate and gender ratio between studies were comparable. Some differences were seen regarding pathological nodal stage and age of patients. Our results revealed that pathological positive nodes and pathological T3/4 tumors were significantly associated with worse survival (Table 1 and Figure 2). Among patients with pathological positive nodes and pathological T3/4 tumors, the 5-year OS and DFS were $37.5 \%$ and $60.9 \%, 41.7 \%$ and $61.8 \%$, respectively. These poor results for high-risk subgroup suggest that more aggressive approach is needed for such a patient. Proportion of patient included in CRAB study with pathological negative nodes was lower than in American study (19.7\% vs. $28 \%) .{ }^{10}$ This fact could affected poorer survival results in CRAB study. Other studies have also shown that pathologic nodal status may represent a superior predictor of better survival for patients with LARC. ${ }^{21,22}$

Furthermore, patient characteristics (sex and age) predict survival. In the largest analysis examining the impact of demographic characteristics on the survival of patients with rectal cancer, older age and male gender are associated with worse cancer-specific and OS. ${ }^{23}$ One of the possible causes for our worse OS could be in the older population that was included in the CRAB study compared to American study (median age with range; 60 years [31-80] vs. 51 years [35-72]). ${ }^{10}$

A comparison between neoadjuvant singleagent capecitabine CRT and the current study revealed promising oncologic outcome with adding bevacizumab in the standard neoadjuvant treatment of LARC in Slovenia (5-year OS, DFS and LC; $61.4,52.4 \%$ and 87.4 vs. $72.2,75.6$ and $92.4 \%){ }^{24}$ Thus, this difference is not statistically significant and we cannot state with certainty that the improvement depends only on adding bevacizumab to standard neoadjuvant capecitabine-based CRT due to differences in the prescribed radiation dose. In CRAB study we used additionally concomitant radiation boost for which it is known that might have a positive effect on OS. ${ }^{25}$ 
TABLE 3. Health related quality of life analysis: Comparisons of mean scores with standard deviations (SD) before and 1 year after completed treatment for all scales of EORTC QLQ-C30 and EORTC QLQ-CR38

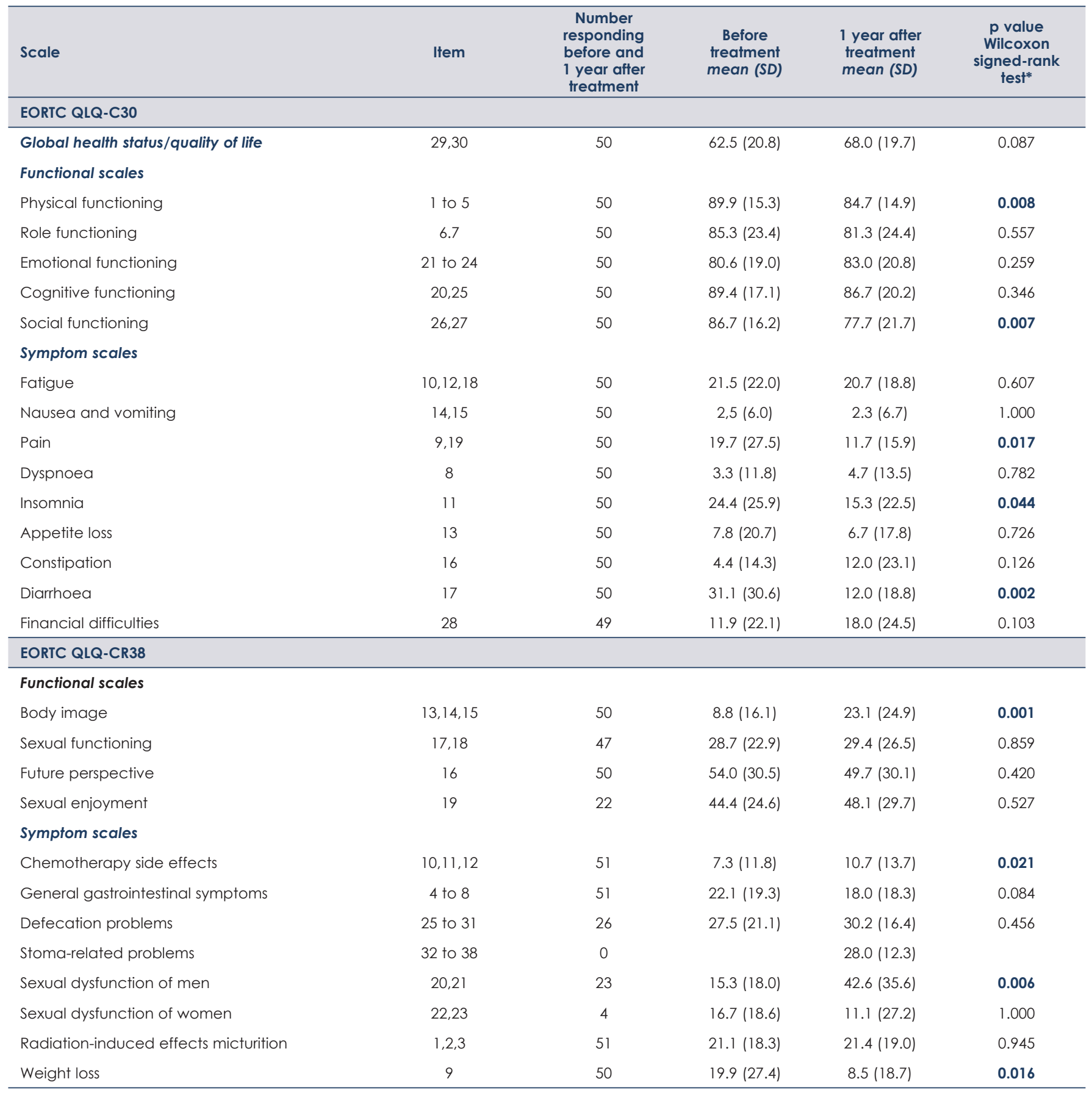

*statistically significant values $(p<0.050)$ are bolded

\section{Late toxicities}

Bevacizumab may cause severe late side effects in metastatic setting but late side effects are relatively rare. ${ }^{26}$ Most often described bevacizumab related late adverse events are spontaneous intestinal per- foration and delayed anastomotic leak..$^{27,28}$ The addition of bevacizumab to standard CRT could be one of the possible causes for a higher proportion of fistulas in our study.

Only few studies analyzed the incidence of the late anastomotic leakage after low anterior resection 
(LAR). Delayed anastomotic leakages that develop after 30 days after surgery are not uncommon. The incidence is between $0.3 \%$ and $9.8 \% .^{29-35}$ It is not clear whether early and late anastomotic leakage after LAR are different entities because some patients with late anastomotic leakage may show uneventful postoperative clinical recovery. ${ }^{29}$ Late leakages more frequently involve the fistula type (22-100\%) than early postoperative leakage. ${ }^{29-32,34,35}$ Pelvic abscess and anastomotic-vaginal fistula were the most common causes of delayed complications. ${ }^{35}$

In our study only one female patient developed anastomotic vaginal fistula 12 months after rectal surgery. In the two studies with higher number of female patients included, the overall rate of anastomotic-vaginal fistula after LAR was higher, 3\% (11/371) and 5.1\% (20/390). ${ }^{36,37}$ Anastomotic-vaginal fistulas in both studies were diagnosed mostly late, on median postoperative day 83 (15-766) in the first study and 25 (5-172) in the second. Despite that only $3 \%$ of the female patients in the first study were treated with preoperative $\mathrm{ChT}$ and no one with RT, pre-op ChT was one of the independent risk factor for anastomotic-vaginal fistula formation. ${ }^{36}$ In the second study, risk factors for anastomotic-vaginal fistula were preoperative radiotherapy, anastomosis $<5 \mathrm{~cm}$ above the anal verge and cancer stage IV. ${ }^{37}$

The incidence of the delayed anastomotic leakage in present study was high $(13.1 \%)$, but it is important that only three fistulas were diagnosed before 5 years of follow up. Comparing the incidence of the delayed anastomotic leakage in our study with other studies is controversial. Follow up in our study was the longest than it was in other published studies. The longest interval between the surgery and fistula formation we found in the literature was 5.7 years and in our study 7.25 years. $\mathrm{RT}$ and ChT were identified as an independent risk factor for the development of the late anastomotic complications. ${ }^{32,34,35}$ In all published studies patients with very mixed type of neo-adjuvant and adjuvant treatment or without treatment were included. On the other side, in our study all patients were treated with neo-adjuvant CRT. According to previous facts, it is important that we pay attention to the appearance of early signs of the fistula during follow up in the patient previous treated with bevacizumab even several years after the completion of treatment.

\section{Health related QOL}

The global health status (mean 68), which refers to the general assessment of the health and qual- ity of life of the last seven days, was similar over time, and also comparable to the Slovenian general (mean 71.1) and CRC patients (mean 68.3)..$^{38,39}$

The values of social and physical functioning indicated by patients were significantly influenced by the time between diagnosis and 1 year after treatment indicating a high incidence of problems in this area. On the other hand, 1 year after treatment patients reported less pain, insomnia and diarrhea. These results are consistent with QLQ-C30 scores of Slovenian CRC patients after surgical treatment. ${ }^{39}$ However, compared above mentioned items with the general Slovenian population, patients 1 year after treatment report more diarrhea, lower physical and social functioning, but less pain and insomnia. The population-based QOL reference values should be taken into account in the interpretation of disease progress and treatment effects. For Slovenian general population it was shown that gender, age and self-rated social class are important confounders in the QOL scores descriptions. ${ }^{38}$

Our long-term trends in longitudinal QOL are in agreement with study published from Couwenberg et al. including rectal cancer patients treated with CRT and surgery. ${ }^{20}$ They demonstrated that treatment of rectal cancer has larger impact on QOL decline within 3-6 months after the start of treatment, but still gradually improves within 1 year after treatment. Moreover, within two years all scores normalize towards pretreatment levels, although compared to general population lower functioning, more insomnia and fatigue persist for more than 2 years from diagnosis.

Based on QLQ-CR38 significantly more ChT side effects and sexual dysfunction of men were observed after 1 year of treatment. However, body image has improved and patients reported less weight loss. Male sexual dysfunction is common and remains high after multimodality treatment for rectal cancer, more precisely, surgical nerve damage remain the main cause. ${ }^{40}$ Patients treated with well-defined and standardized technique of total mesorectal excision together with minimally invasive techniques experienced less sexual dysfunction compared to conventional surgery. ${ }^{41}$ In addition, laparoscopic resection contributes more to the maintenance of the nerves..$^{40}$

\section{Limitations}

The main limitations of this study include the single center design of the study with small number of patients, which limits statistical power. To assess 
the QOL we have chosen a QLQ-CR38 and not revised successor QLQ-CR29, because the latter was published later in 2007. Consequently, our results are less comparable to others that have been used QLQ-CR29.

\section{Conclusions}

The optimal treatment strategy for patients with LARC is still controversial. Neoadjuvant bevacizumab with standard CRT in LARC is acceptable strategy. Further studies of its effect on better longterm outcome are warranted. Patients with LARC with high risk factors, such as positive pathological lymph nodes and high pathological $\mathrm{T}$ stage, deserve more aggressive treatment in the light of improving long-term survival results. While advances in multimodality treatment of CRC are enormous, some of the QOL aspects and long-term safety are often not published and inadequately discussed with patients. LARC treatment may no longer be standardized, but adjusted to the wishes, needs and characteristics of an individual patient.

\section{References}

1. Bray F, Ferlay J, Soerjomataram I, Siegel RL, Torre LA, Jemal A. Global cance statistics 2018 : GLOBOCAN Estimates of incidence and mortality worldwide for 36 cancers in 185 countries. CA Cancer J Clin 2018; 68: 394-424. doi: 10.3322/caac. 21492

2. Zadnik V, Primic Zakelj M, Lokar K, Jarm K, Ivanus U, Zagar T. Cancer burden in Slovenia with the time trends analysis. Radiol Oncol 2017; 51: 47-55. doi: 10.1515/raon-2017-0008

3. Ludmir EB, Palta M, Willett CG, Czito BG. Total neoadjuvant therapy for rectal cancer: An emerging option. Cancer 2017; 123: 1497-506. doi: 10.1002/ cncr.30600

4. Sauer R, Becker H, Hohenberger W, Rödel C, Wittekind C, Fietkau R, et al. Preoperative versus postoperative chemoradiotherapy for rectal cancer. $N$ Engl J Med 2004; 351: 1731-40. doi: 10.1056/NEJMoa040694

5. Sauer R, Liersch T, Merkel S, Fietkau R, Hohenberger W, Hess C, et al. Preoperative versus postoperative chemoradiotherapy for locally advanced rectal cancer: results of the German CAO/ARO/AIO-94 randomized phase III trial after a median follow-up of 11 years. J Clin Oncol 2012; 30: 1926-33. doi: 10.1200/JCO.2011.40.1836

6. Mody K, Baldeo C, Bekaii-Saab T. Antiangiogenic therapy in colorectal cancer. Cancer J 2018; 24: 165-70. doi: 10.1097/PPO.0000000000000328

7. Nieder C, Wiedenmann N, Andratschke NH, Astner ST, Molls M. Radiation therapy plus angiogenesis inhibition with bevacizumab: rationale and initial experience. Rev Recent Clin Trials 2007; 2: 163-8. doi: $10.2174 / 157488707781662733$

8. Willett CG, Boucher Y, Duda DG, di Tomaso E, Munn LL, Tong RT, et al. Surrogate markers for antiangiogenic therapy and dose-limiting toxicities for bevacizumab with radiation and chemotherapy: continued experience of a phase I trial in rectal cancer patients. J Clin Oncol 2005; 23: 8136-9. doi: 10.1200/JCO.2005.03.5881

9. Fornaro L, Caparello C, Vivaldi C, Rotella V, Musettini G, Falcone A, et al. Bevacizumab in the pre-operative treatment of locally advanced recta cancer : a systematic review. World J Gastroenterol 2014; 20: 6081-91. doi: 10.3748/wjg.v20.i20.6081
10. Willett CG, Duda DG, Di Tomaso E, Boucher Y, Ancukiewicz M, Sahani D V, et al. Efficacy, safety, and biomarkers of neoadjuvant bevacizumab, radiation therapy, and fluorouracil in rectal cancer: multidisciplinary phase II study. $J$ Clin Oncol 2009; 27: 3020-6. doi: 10.1200/JCO.2008.21.1771

11. García M, Martinez-Villacampa M, Santos C, Navarro V, Teule A, Losa F, et al. Phase II study of preoperative bevacizumab, capecitabine and radiotherapy for resectable locally-advanced rectal cancer. BMC Cancer 2015; 15: 59. doi: 10.1186/s12885-015-1052-0

12. Sprangers MA, Te Velde A, Aaronson NK. The construction and testing of the EORTC colorectal cancer-specific quality of life questionnaire module (QLOCR38). Eur J Cancer 1999; 35: 238-47. doi: 10.1016/S0959-8049(98)00357-8

13. Gujral S, Conroy T, Fleissner C, Sezer O, King PM, Avery KN, et al. Assessing quality of life in patients with colorectal cancer : an update of the EORTC quality of life questionnaire. Eur J Cancer 2007; 43: 1564-73. doi: 10.1016/j. ejca.2007.04.005

14. Ganesh V, Agarwal A, Popovic M, Cella D, Mcdonald R, Vuong S, et al Comparison of the FACT-C, EORTC QLQ-CR38, and QLQ-CR29 quality of life questionnaires for patients with colorectal cancer: a literature review. Support Care Cancer 2016; 24: 3661-8. doi: 10.1007/s00520-016-3270-7

15. Velenik V, Ocvirk J, Mušič $M$, Bračko $M$, Anderluh F, Oblak I, et al. Neoadjuvant capecitabine, radiotherapy, and bevacizumab (CRAB) in locally advanced rectal cancer: results of an open-label phase II study. Radiat Oncol 2011; 6: 105. doi: 10.1186/1748-717X-6-105

16. Aaronson N, Ahmedzai S, Bergman B, Bullinger M, Cull A, Duez N, et al. The European organisation for research and treatment of cancer QLQ-C30: a quality-of-life instrument for use in international clinical trials in oncology. $J$ Natl Cancer Inst 1993; 85: 365-76. doi: 10.1093/jnci/85.5.365

17. Fayers P, Aaronson N, Bjordal K, Groenvold M, Curran D, Bottomley A. EORTC QLQ-C3O scoring manual. 3rd Edition. Brussels: European Organisation for Research and Treatment of Cancer; 2001.

18. Velenik V, Anderluh F, Oblak I, Strojan P, Zakotnik B. Capecitabine as a radiosensitizing agent in neoadjuvant treatment of locally advanced resectable rectal cancer: prospective phase II trial. Croat Med J 2006; 47: 693-700. PMID: 17042060

19. Gasparini G, Torino F, Ueno T, Cascinu S, Troiani T, Ballestrero A, et al. A phase II study of neoadjuvant bevacizumab plus capecitabine and concomitant radiotherapy in patients with locally advanced rectal cancer. Angiogenesis 2012; 15: 141-50. doi: 10.1007/s10456-011-9250-0

20. Couwenberg AM, Burbach JPM, van Grevenstein WMU, Smits AB, Consten ECJ, Schiphorst AHW, et al. Effect of neoadjuvant therapy and rectal surgery on health-related quality of life in patients with rectal cancer during the first 2 years after diagnosis. Clin Colorectal Cancer 2018; 17: 499-512. doi 10.1016/j.clcc.2018.03.009

21. Kim NK, Baik SH, Seong JS, Kim H, Roh JK, Lee KY, et al. Oncologic outcomes after neoadjuvant chemoradiation followed by curative resection with tumor-specific mesorectal excision for fixed locally advanced - impact of postirradiated pathologic downstaging on local reccurrence and survival. Ann Surg 2006; 244: 1024-30. doi: 10.1097/01.sla.0000225360.99257.73

22. Hernandez JM, Clark W, Weber J, Fulp WJ, Lange L, Shibata D. The impact of pathologic nodal status on survival following neoadjuvant chemoradiation for locally advanced rectal cancer. Int J Color Dis 2014; 29: 1061-8. doi: 10.1007/s00384-014-1917-8

23. Berger MD, Yang D, Sunakawa Y, Zhang W, Ning Y, Matsusaka S, et al. Impact of sex, age and ethnicity/race on the survival of patients with rectal cancer in the United States from 1988 to 2012. Oncotarget 2016; 7: 53668-78. doi: 10.18632/oncotarget.10696

24. Velenik V, Oblak I, Anderluh F. Long-term results from a randomized phase II trial of neoadjuvant combined-modality therapy for locally advanced rectal cancer. Radiat Oncol 2010; 5: 88. doi: 10.1186/1748-717x-5-88

25. Badakhshi H, Ismail M, Boskos C, Zhao K, Kaul D. The role of concomitant radiation boost in neoadjuvant chemoradiotherapy for locally advanced rectal cancer. Anticancer Res 2017; 37: 3201-5. doi: 10.21873/anticanres.11681

26. O'Hare T, McDermott R, Hannon R. Late anastomotic breakdown with bevacizumab in colorectal cancers, a case-based review. Ir J Med Sci 2018; 187: 333-6. doi: 10.1007/s11845-017-1676-y

27. Borzomati D, Nappo G. Infusion of bevacizumab increases the risk of intestinal perforation: results on a series of 143 patients consecutively treated. Updat Surg 2013; 65: 121-4. doi: 10.1007/s13304-013-0207-2 
28. Machida E, Miyakura Y, Takahashi J, Tamaki S, Ishikawa H, Hasegawa F, et al. Bevacizumab is associated with delayed anastomotic leak after low anterior resection with preoperative radiotherapy for rectal cancer: a case report. Surg Case Rep 2019; 5: 14. doi: 10.1186/s40792-019-0573-1

29. Floodeen H, Hallböök O, Rutegård J, Sjödahl R, Matthiessen P. Early and late symptomatic anastomotic leakage following low anterior resection of the rectum for cancer: are they different entities? Color Dis 2013; 15: 334-40. doi: 10.1111/j.1463-1318.2012.03195.x

30. Hyman N, Manchester TL, Osler T, Burns B, Cataldo PA. Anastomotic leaks after intestinal anastomosis: it's later than you think. Ann Surg 2007; 245: 254-8. doi: 10.1097/01.sla.0000225083.27182.85

31. Iwamoto M, Kawada K, Hida K, Hasegawa S, Sakai Y. Delayed anastomotic leakage following laparoscopic intersphincteric resection for lower rectal cancer: report of four cases and literature review. World J Surg Oncol 2017; 15: 143. doi: 10.1186/s12957-017-1208-2

32. Lim S, Yu CS, Kim CW, Yoon YS, Park IJ, Kim JC. Late anastomotic leakage after low anterior resection in rectal cancer patients: clinical characteristics and predisposing factors. Color Dis 2016; 18: 135-40. doi: 10.1111/codi.13300

33. Matthiessen P, Lindgren R, Hallböök O, Rutegård J, Sjödahl R, Study GRCT on DS. Symptomatic anastomotic leakage diagnosed after hospital discharge following low anterior resection for rectal cancer. Color Dis 2010; 12: 82-7. doi: 10.1111/j.1463-1318.2009.01938.x

34. Morks AN, Ploeg RJ, Sijbrand Hofker H, Wiggers T, Havenga K. Late anastomotic leakage in colorectal surgery : a significant problem. Color Dis 2013; 15: 271-5. doi: 10.1111/codi.12167

35. Shin US, Kim CW, Yu CS, Kim JC. Delayed anastomotic leakage following sphincter-preserving surgery for rectal cancer. Int J Color Dis 2010; 25: 8439. doi: 10.1007/s00384-010-0938-1

36. Watanabe J, Ota M, Kawaguchi D, Shima H, Kaida S, Osada S, et al. Incidence and risk factors for rectovaginal fistula after low anterior resection for rectal cancer. Int J Color Dis 2015; 30: 1659-66. doi: 10.1007/s00384-015-2340-5

37. Matthiessen P, Hansson L, Sjödah I, Rutegård J. Anastomotic-vaginal fistula ( AVF) after anterior resection of the rectum for cancer - occurrence and risk factors. Color Dis 2010; 12: 351-7. doi: 10.1111/j.1463-1318.2009.01798.x

38. Velenik V, Šečerov-Ermenc A, But-Hadžić J, Zadnik V. Health-related quality of life assessed by the EORTC QLQ-C30 questionnaire in the general slovenian population. Radiol Oncol 2017; 51: 342-50. doi: 10.1515/ raon-2017-0021

39. Grosek J, Novak J, Kitek K, Bajrič A, Majdič A, Košir JA. Health-related quality of life in Slovenian patients with colorectal cancer: a single tertiary care center study. Radiol Oncol 2019; 53: 231-7. doi: 10.2478/raon-2019-0015

40. Nagpal K, Bennett N. Colorectal surgery and its impact on male sexual function. Curr Urol Rep 2013; 14: 279-84. doi: 10.1007/s11934-013-0341-x

41. Chew $M$, Yeh $\mathrm{Y}$, Lim E, Seow-Choen F. Pelvic autonomic nerve preservation in radical rectal cancer surgery: changes in the past 3 decades. Gastroenterol Rep 2016; 4: 173-85. doi: 10.1093/gastro/gow023 